\title{
Isolated Metastatic Barrett's Adenocarcinoma of the Thoracic Epidural Space Causing Paraplegia
}

\author{
Tomohiro Banno $^{1}$, Tsuyoshi Ohishi ${ }^{1}$, Daisuke Suzuki ${ }^{1}$, Kazufumi Yamamoto ${ }^{1}$, Hiroki Ushirozako ${ }^{1}$, \\ Sho Kobayashi' ${ }^{2}$, Yukihiro Matsuyama ${ }^{2}$ \\ ${ }^{1}$ Departments of Orthopaedic Surgery, Enshu Hospital, Hamamatsu, Shizuoka, Japan; ${ }^{2}$ Departments of Orthopaedic Surgery, Hama- \\ matsu University School of Medicine, Hamamatsu, Shizuoka, Japan. \\ Email: tomo-ban.0311@s8.dion.ne.jp
}

Received January $17^{\text {th }}, 2013$; revised March $1^{\text {st }}$, 2013; accepted March $18^{\text {th }}, 2013$

\begin{abstract}
Barrett's adenocarcinoma is an esophageal cancer arising from Barrett's esophagus. In this report, a metastatic epidural tumor from Barrett's adenocarcinoma with paraplegia that required surgical treatment is documented. A 50-year-old man who had had a resection of the distal esophagus due to Barrett's adenocarcinoma six month before presented with progressive weakness and numbness in lower limbs with urinary disturbance. Magnetic resonance imaging of the thoracic region revealed compression of the spinal cord at the T3-4 level by a dorsally located epidural mass. No signal changes were detected in vertebrae. Since the paraplegia and urinary disturbance were rapidly progressing, laminectomy and tumor resection was performed. Postoperatively, neurological function improved immediately and there was no recurrence of spinal region. This is the first report on an isolated metastatic Barrett's adenocarcinoma of the thoracic epidural space.
\end{abstract}

Keywords: Barrett’s Adenocarcinoma; Metastasis; Isolated Epidural Tumor

\section{Introduction}

Barrett's esophagus, which is defined as intestinal metaplasia in the distal esophagus, is considered one of the risk factors of esophageal adenocarcinoma [1]. Most cases of esophageal carcinoma are pathologically squamous cell carcinoma among Japanese, however adenocarcinoma derived from Barrett's esophagus called Barrett's adenocarcinoma is increasing in number recently [2]. Although several case reports of the metastatic cancer in the epidural space have been documented, that derived from esophageal carcinoma is rare. To our knowledge, no cases of epidural metastasis without bone involvement from Barrett's adenocarcinoma have been reported in the literature. We present here a case of metastasis to the thoracic epidural space from adenocarcinoma in Barrett's esophagus that required surgical intervention due to progressive paraplegia.

\section{Case Report}

A 50-year-old man presented with a 2-day history of progressive weakness and numbness of lower limbs. Six months previously, he had a resection of the distal esophagus due to adenocarcinoma in Barrett's esophagus. As metastatic para-aortic lymph nodes were observed during operation, complete lymphadenectomy was per- formed. No other organ metastasis or distant lymph nodes metastasis was detected by computed tomography of the chest-abdominal region. He was categorized as stage 4 according to the TNM stage classification. He could not walk nor stand from a squatting position. He complained of difficulty urinating in the morning. Neurological examination revealed that muscle motor test of lower limbs was 2/5 in iliopsoas, 2/5 in quadriceps, 3/5 in tibialis anterior, 3/5 in extensor hallucis longus, and $3 / 5$ in gastrocnemius bilaterally. Severe deterioration of sense was manifested under the level of umbilicus. Deep tendon reflexes of both lower extremities were hypereflexia and Babinski response was obtained bilaterally. Plain radiographs and computed tomography of the thoracic spine revealed no signs of bone metastasis including bone destruction or erosion. Magnetic resonance imaging (MRI) of the thoracic spine revealed compression of the spinal cord at the T3-4 level by a dorsally located epidural mass with isointensity and enhanced on T1weighted MR images and increased signal intensity on T2-weighted MRI (Figure 1). No signal changes were detected in vertebrae. Laminectomy of T3 and T4 and tumor resection was performed under general anesthesia since the paraplegia and urinary disturbance were rapidly progressing. The tumor was identified on the dorsal side of dura matter after laminectomy. No adhesion around 
the tumor was observed and the tumor was easily excised en bloc (Figure 2). Histopathological examinations confirmed a metastasic adenocarcinoma arising from the thoracic epidural space (Figure 3). Postoperatively, motor dysfunction and dysuria improved immediately and adjuvant chemotherapy was started. On follow-up MRI at 3-month postoperatively, the epidural mass disappeared and no other metastasis were detected although intramedullary increased signal on T2-weighted MRI appeared that was considered as a spinal cord injury (Figure 4). At 3 month after the operation, he was ambulatory with a walking aid. But he died 7 month after the operation due to lung metastasis. No recurrence of spinal region was observed on pathological anatomy.
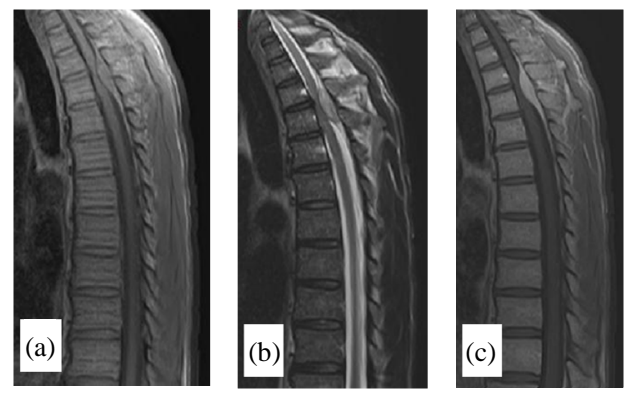

Figure 1. Sagittal image of thoracic spine. Spinal cord was compressed by the dorsally located epidural tumor at the T3-4 level with isointensity and enhanced on T1-weighted MR images ((a) and (c)) and increased signal intensity on T2-weighted MRI (b).

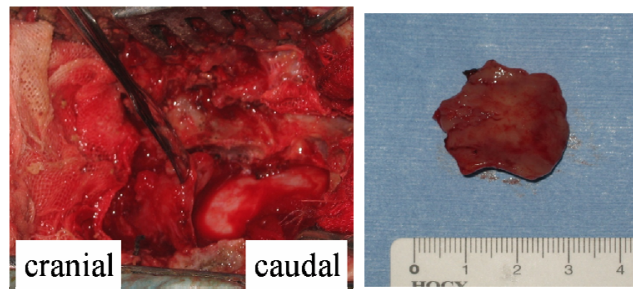

Figure 2. An intraoperative photograph (Left). The epidural tumor existed on dura matter without adhesion and was easily excised en bloc (Right).

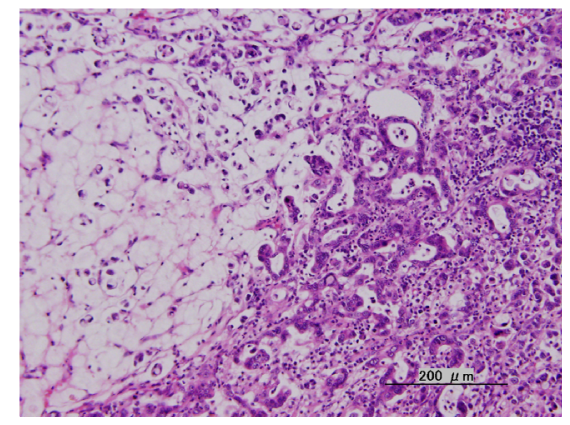

Figure 3. Histological appearance of epidural mass. Atypical epithelioglandular cells spread and formed an irregular glandular structure compatible with metastatic adenocarcinoma.

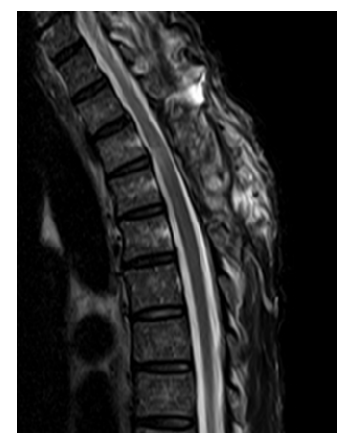

Figure 4. Axial T2-weighted image of the thoracic spine examined at 3 months after operation. The epidural tumor was excised entirely without any local reoccurrences.

\section{Discussion}

Barrett's adenocarcinoma was defined as a tumor located in the tubular part of the esophagus and arising within the zone of Barrett's metaplasia [3]. Barrett's esophagus is considered to be strongly associated with esophageal adenocarcinoma [4]. The relative risk of adenocarcinoma among patients with Barrett's esophagus was 10 - 40 times that of the general population, and the incidence rate was 1.2 to 5.0 per 1000 person-year from previous studies [5-7]. Adenocarcinoma accounts for almost half of esophageal cancers among Europeans and Americans, however the majority of esophageal cancers among Japanese are squamous cell carcinoma [2,8]. Although the incidence rate for adenocarcinoma out of esophageal carcinomas among Japanese was only $1 \%-2 \%$, it is now increasing [2]. Spinal epidural metastasis occurs in 5\% $10 \%$ of all cancer patients. In particular, lung, breast, prostate, kidney, and lymphs are common as the primary site [9]. Spinal epidural metastasis of esophageal carcinoma is rare and the incident rate is about $1 \%$ [10-12]. Among metastatic tumors in the epidural space, approximately $60 \%$ are located in the thoracic spine and $30 \%$ are located in the lumbosacral spine [9]. To our knowledge, no cases of isolated epidural metastatic tumor without bone involvement from Barrett's adenocarcinoma have been reported in the literature. Most metastatic epidural tumors arise in vertebral bodies or pedicles first, followed by invasion of the epidural space. An isolated epidural tumor without bone involvement might be caused by a direct hematogenous dissemination via radicular arteries or vertebral venous plexus from a primary site that was not apparent during the primary operation $[4,13,14]$. Good neurological recovery resulted after the tumor was resected en bloc and the patient was ambulatory again postoperatively. The prognosis of advanced Barrett's adenocarcinoma is generally poor although a good prognosis is expected in early stage cases. The five-year survival rate for a patient without lymph node metastasis is $85.3 \%$, whereas that with distant lymph node metastasis (stage 4) is 0\% [1]. In this case, 
though the patient was died 13 months after the initial operation, there was no recurrence of spinal region. Therefore, epidural tumor resection was effective for maintaining his daily living

\section{Conclusion}

An isolated epidural metastatic tumor without bone involvement is rare and thought to be caused by a direct hematogenous dissemination via radicular arteries or vertebral venous plexus from a primary site; indeed, metastatic para-aortic lymph nodes were observed during the first operation, and the patient was categorized as stage 4 according to the TNM stage classification. In this case of isolated metastatic epidural tumor from Barrett's adenocarcinoma, no adhesion around the tumor was observed and it was easily excised en bloc, therefore good neurological recovery should be obtained postoperatively.

\section{REFERENCES}

[1] T. Lerut, W. Coosemans, D. Van Raemdonck, B. Dillemans, P. De Leyn, J. M. Marnette and K. Geboes, “ Surgical Treatment of Barrett's Carcinoma,” The Journal of Thoracic Cardiovascular Surgery, Vol. 107, No. 4, 1994, pp. 1059-1065.

[2] M. Hongo and T. Shoji, "Epidemiology of Reflux Disease and CLE in East Asia,” Journal of Gastroenterology, Vol. 38, No. 15, 2003, pp. 25-30.

[3] B. C. Morson and J. R. Belcher, "Adenocarcinoma of Oesophagus and Ectopic Gastric Mucosa,” British Journal of Cancer, Vol. 6, No. 2, 1953, pp. 127-130. doi:10.1038/bjc.1952.14

[4] O. V. Batson, "The Function of the Vertebral Veins and Their Role in the Spread of Metastases," Annals of Surgery, Vol. 112, No. 1, 1940, pp. 138-149. doi:10.1097/00000658-194007000-00016

[5] F. Hvid-Jensen, L. Pedersen, A. M. Drewes, H. T. Søren- sen and P. Funch-Jensen, "Incidence of Adenocarcinoma among Patients with Barrett's Esophagus,” New England Journal of Medicine, Vol. 365, No. 15, 2011, pp. 13751383. doi:10.1056/NEJMoa1103042

[6] L. Murray, P. Watson, B. Johnston, J. Sloan, I. M. Mainie and A. Gavin, "Risk of Adenocarcinoma in Barrett's Oesophagus,” BMJ, Vol. 327, No. 7414, 2003, pp. 534-535. doi:10.1136/bmj.327.7414.534

[7] M. Solaymani-Dodaran, R. F. Logan, J. West, T. Card and C. Coupland, "Risk of Oesophagus Cancer in Barrett's Oesophagus and Gastro-Oesophageal Reflex," Scandinavian Journal of Gastroenterology, Vol. 39, No. 7, 2004, pp. 680-685. doi:10.1080/00365520410004802

[8] S. S. Devesa, W. J. Blot and J. F. Fraumeni, "Changing Patterns in the Incidence of Esophageal and Gastric Carcinoma in the United States,” Cancer, Vol. 83, No. 10, 1998, pp. 2049-2053.

doi:10.1002/(SICI)1097-0142(19981115)83:10<2049::AI D-CNCR1>3.0.CO;2-2

[9] M. Mut, D. Schiff and M. E. Shafferey, "Metastatic to Nerveous System: Spinal Epidural and Intramedullsry Metastasis,” Journal of Neuro-Oncology, Vol. 75, No. 1, 2005, pp. 43-56. doi:10.1007/s11060-004-8097-2

[10] J. Brice and W. Mckissock, "Surgical Treatment of Malignant Extradural Spinal Tumors," British Medical Journal, Vol. 1, No. 5446, 1965, pp. 1339-1342. doi:10.1136/bmj.1.5446.1341

[11] H. O. Chade, "Metastatic Tumors of the Spine and Spinal cord," Handbook of Clinical Neurology, Vol. 20, No. 2, 1976, pp. 415-433.

[12] R. L. Wright, "Malignant Tumors in the Spinal Extradural Space: Results of Surgical Treatment," Annals of Surgery, Vol. 157, No. 2, 1963, pp. 227-231.

[13] M. Rodoriguez and R. P. Dinapoli, "Spinal Cord Compression with Special Reference to Metastatic Epidural Tumors,” Mayo Clinic Proceedings, Vol. 55, No. 7, 1980, pp. 442-448.

[14] K. Shibasaki, C. G. Harper and G. M. Bedbrock, "Vertebral Metastases and Spinal Cord Compression," Paraplegia, Vol. 21, No. 1, 1983, pp. 47-61. 